\title{
AiMT
}

Advances in Military Technology

Vol. 13, No. 1 (2018), pp. 119-131

ISSN 1802-2308, eISSN 2533-4123

DOI 10.3849/aimt.01230

\section{Critical Crack Size Investigation Method for a Land Launcher}

\author{
A. Yetgin ${ }^{1 *}$, B. Acar ${ }^{1}$ and S. Kadıoğlu ${ }^{2}$ \\ ${ }^{1}$ Research \& Development Department, Roketsan, Turkey \\ ${ }^{2}$ Department of Mechanical Engineering, Middle East Technical University, Turkey
}

The manuscript was received on 9 January 2018 and was accepted after revision for publication on 12 May 2018.

\begin{abstract}
:
Structural integrity evaluation of military systems is vital in such applications as rocket launchers. In safe life design, for a certain operational life the system is ensured to function well and no failure would occur even in the presence of some imperfections or flaws. This paper considers the fracture analysis as part of the safe life design approach that is used in the design of a rocket launcher. A methodology based on sub modelling technique is introduced. It was made sure that unstable crack growth would not occur upto certain crack sizes. Subsequently, after actual manufacturing of the launcher, critical locations of welds in the system were checked for presence of any cracks after repeated firing loads. After these controls no cracks were detected due to operational conditions until the time when this document was prepared.
\end{abstract}

\section{Keywords:}

fracture mechanics, weld, finite element, launcher, structural integrity

\section{Introduction}

Multi barrel rocket launchers contain multiple rockets and are used for artillery support in the army. They are usually agile and need little preparation time for firing. The launchers can be used with both guided and unguided munitions. Rocket launcher systems, which are unguided are used to cover a certain region by heavy artillery fire instead of single accurate shots. In Fig. 1 a photograph of a land rocket launcher vehicle can be seen.

The launcher systems are needed to be used many times in the field, thus the system is subjected to repetitive loading. Therefore, it is crucial to determine the loads on the system very accurately and to evaluate the structural integrity precisely. It would

\footnotetext{
* Corresponding author: Research \& Development Department, Roketsan, Ankara, Turkey. Phone+903128605500,E-mail: ayetgin@roketsan.com.tr
} 
be catastrophic if any malfunction of the system occurs during field operations. Such an event puts personnel and mission at great risk.

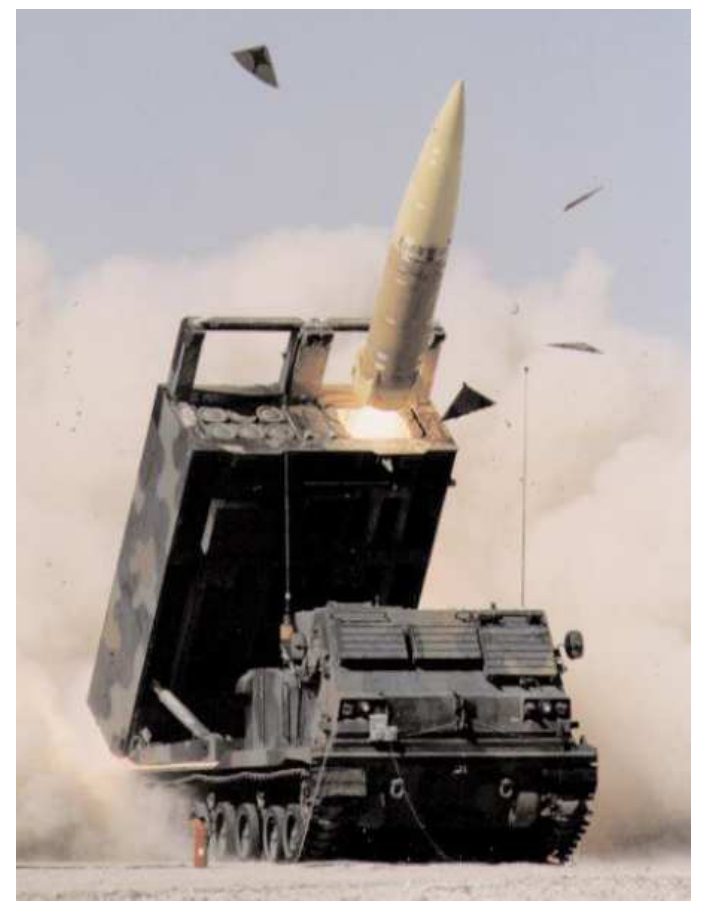

Fig. 1 A land rocket launcher vehicle [1]

One possible failure mode for system components is fracture, resulting from crack like flaws. Since it is almost impossible to build a structure without any such flaws, it is important to have a design that endures them up to a certain level. At this point fracture mechanics comes into picture with the use of which, new damage-tolerant designs have become available [2]. Structure's integrity is now dependent on applied stress, flaw size and fracture toughness of the material. Geometric discontinuities in the body cause an increased stress field around them. This situation causes local stresses to reach material's strength limit even under modest nominal loadings [2]. Fracture limit of the structure can be lower than the plastic limit of the material. It is therefore important to include fracture mechanics analysis in the design cycle.

Critical systems like rocket launchers require safe life design approach. In safe life design, for a certain operational life the system is ensured to function well and no failure would occur. After the expected service period, the system is either replaced or repaired. The life of the system is determined through an accurate analysis and testing. In safe life design philosophy, since the system is designed not to fail during service period, accurate analysis and thorough testing is essential. Fatigue and fracture mechanics are key analysis methods in safe life design approach. The cost of failure of a rocket launcher system during mission may be the loss of personnel or an unaccomplished mission. The chosen design approach must be applied appropriately and the design must allow proper inspection of the system for any indication of failure. Although systems have a predetermined operational life with some safety factor, the system should be checked against for any indication of failure. 
The study presented in this paper is a part of safe life design approach that is used in the design of a multi barrel rocket launcher. Structural integrity of welded connections in a multi barrel launcher system is investigated on a fracture mechanics basis. Material characterization for welded and base materials was performed in order to obtain mechanical properties. Finite element model of the launcher system was built on a global scale by using ABAQUS ${ }^{\circledR}$ [3]. While global model includes all necessary elements such as kinematic and elastic connections in order to simulate the structural and dynamic response of the launcher, weld details are investigated on separate local models. Local models, (i.e. sub models) include finer finite element mesh and global model results were used as boundary conditions for local models. The launcher prototype has been manufactured and tested on the field. Certain measurements were made and compared with finite element model. With validated methodology structural integrity assessment of the structure is done against operational loads and possible defects on the welds.

\section{Methodology}

In order to perform fracture mechanics analysis, a detailed finite element mesh must be generated in the region around a crack. However this cannot be performed for most of the real world problems. Overall response of the structure under external loads is obtained by creating a global model, which does not contain any cracks. This model includes all components that affect the response of the structure. Global models become relatively large since they include many parts and connections. Therefore, level of detail to be modelled in the mesh of the structure is reduced. However, these details do not affect the general deformation and stress field in the structure. Generally local details such as fillets, small holes, rivets or fasteners are omitted. Including all details in global model makes the finite element model too large to handle and increases computing time greatly. Therefore it is reasonable to work with global and sub models. As an example of such an approach, the article by Diamantoudis and Labeas [4] where they considered the stress intensity factor (SIF) calculation for the cracks existing in the pressure vessels can be mentioned. They obtained SIF solutions for different surface crack dimension and locations under various pressure loads. Pursuing this aim they implemented Finite Element Analysis (FEA) technique using ANSYS ${ }^{\circledR}$. Firstly global models without cracks were solved for different configurations and then they used sub-models containing the cracks for SIF calculations. This method is proven to be efficient and accurate instead of modelling the whole model containing cracks. Another relatively more recent study, which is methodwise similar to the current one is the article by Giglio and Manes [5]. They investigated crack growth on helicopter panels on an experimental and FEA basis. In the experiment phase, full scale tests were conducted on an aluminum panel with initial crack under fatigue loads. During the test crack growth and strain measurements are taken on various positions on the panel. In FEA phase, global model of the panel and sub-model containing crack region were created. SIFs are calculated using FEA models and analytical methods and the findings are compared. The crack propagation prediction made by FEA model and experimental results are also compared. The results yielded good agreement.

In sub-models, all necessary details that are omitted in the global model are included. They cover only a limited portion of the whole structure. The boundary conditions in the sub models are obtained from the global model results. In sub-models a finer mesh is applied around the details, which would not be practical to include in 
the global model. Unlimited number of sub-models can be created and analyzed without having to run the whole model again under the same boundary conditions. This allows the designer to investigate different designs and to find solutions in a comparatively less time.

Sub-models are created at locations where the details of the structure need to be investigated. The details examined in this study are welded connections and cracks that are modelled in these connections. In order to model cracks, a solid model should be created. Boundary conditions must be transferred from shell elements of the global to solid elements of the sub-model. (see Fig. 2) Methodology used here can be summarized as follows:

1. Local region of interest is determined.

2. Local region is selected by adding some more regions on the boundaries.

3. Additionally selected regions are left to be modelled with shell elements.

4. Region of interest is modelled using solid elements.

5. Shell to solid coupling is defined between solid and shell parts.

6. Boundary conditions are imported from global shell model to the shell boundary elements.

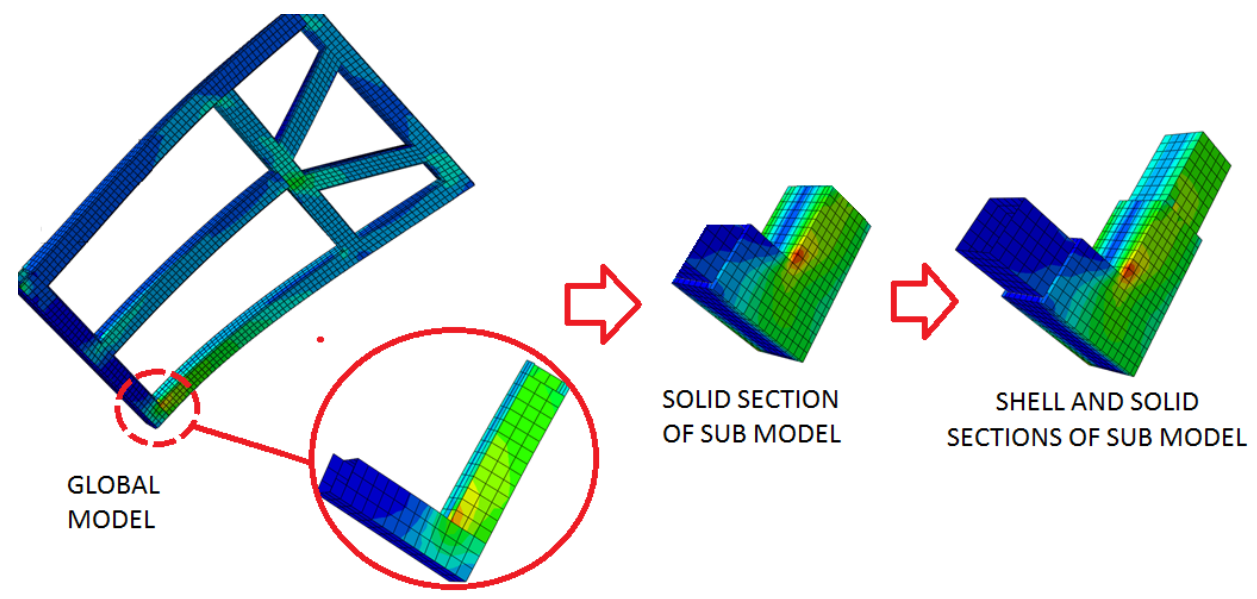

Fig. 2 Sub modelling methodology

\section{Global Model}

Global finite element model includes all sources of non-linearity such as material, geometry and contact. By including necessary elements in the global model, dynamic and structural response of the structure under operational loads are captured well. This allows designers to evaluate their designs more accurately prior to testing. Realistic simulations are obtained in computer environment and hence overall cost and time of design cycle is reduced. An accurate model also gives opportunity to notice unexpected response of the structure if there is any. The launcher system under consideration in this study is shown in Fig. 3. 


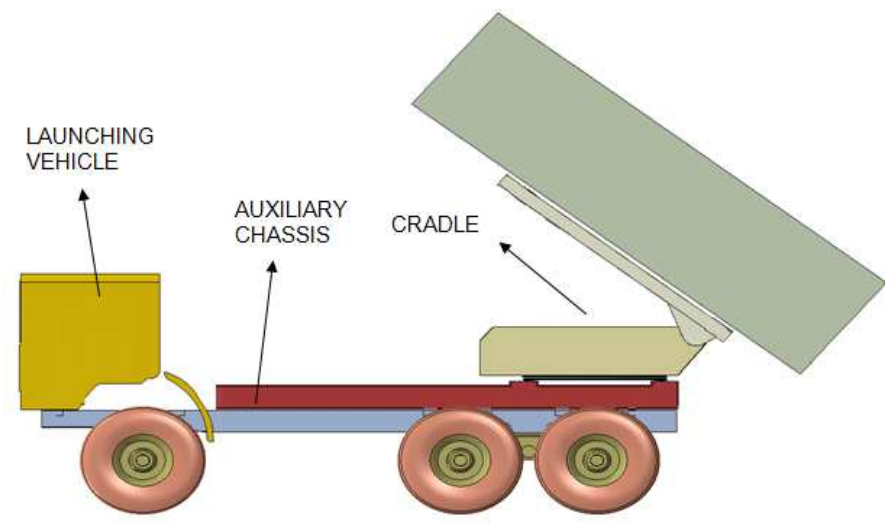

Fig. 3 Launcher system model

Within this launcher system, the focus of interest in this study is the cradle part. When the missiles are launched, this part is subjected to the plume load as well as its own weight and these forces are transmitted to the launcher vehicle. The loading on the cradle is schematically shown in Fig. 4.

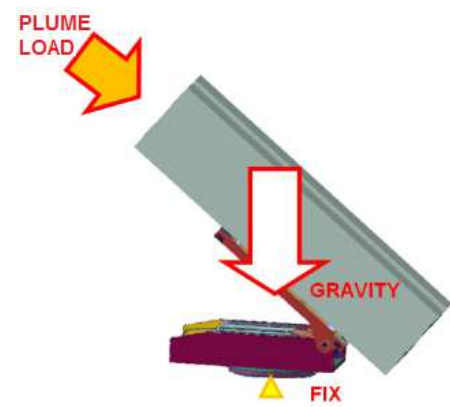

Fig. 4 Loading on the cradle

The global model in Fig. 3, which is being analysed includes structural parts, kinematic connections, elastic springs and weld connections. The majority of the model is constructed using shell elements. Hydraulic pistons are modelled using beam connections. Hinge connections are modelled using appropriate connector definitions. The launcher includes a slewing ring that allows it to turn in azimuth angles. The slewing ring is at the connection of launcher to vehicle. It must withstand transportation and firing loads and allow rotation of cradle with minimum effort.

The slewing ring in the global model is created with a special modelling method. Every ball in the slewing ring is represented by a couple of elastic springs (Fig. 5). These springs carry the load in compression direction as this should be the case. The slewing ring is a major component of cradle finite element model that affects dynamic response. Spring constants for every ball in the slewing ring are defined by a nonlinear curve. For every ball in the ring the same nonlinear spring characteristic is used. This definition allows the balls to carry the load in compression direction only. As it is seen in Fig. 6, the spring exerts force in compression displacement while there is no force under tensile displacement. This is the proper behaviour for a ball in the raceway of slewing ring. 


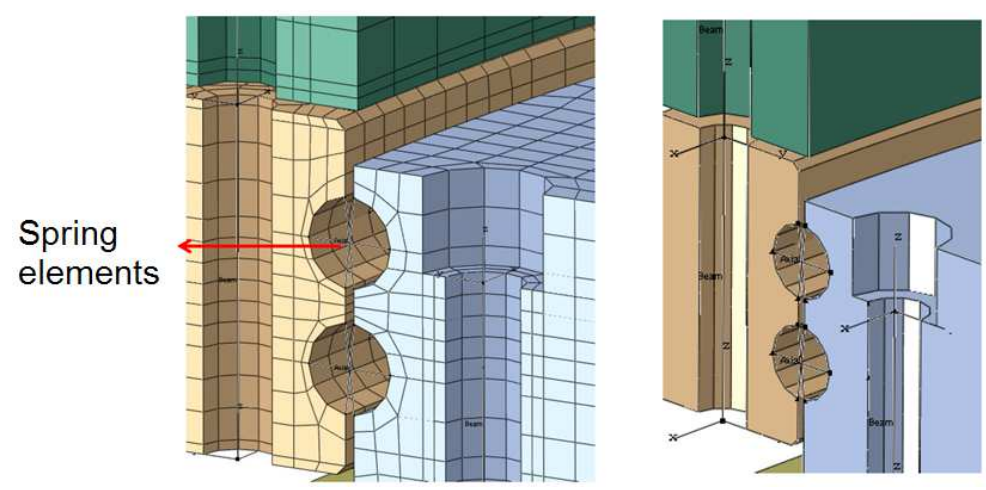

Fig. 5 Spring elements representing slewing ring balls
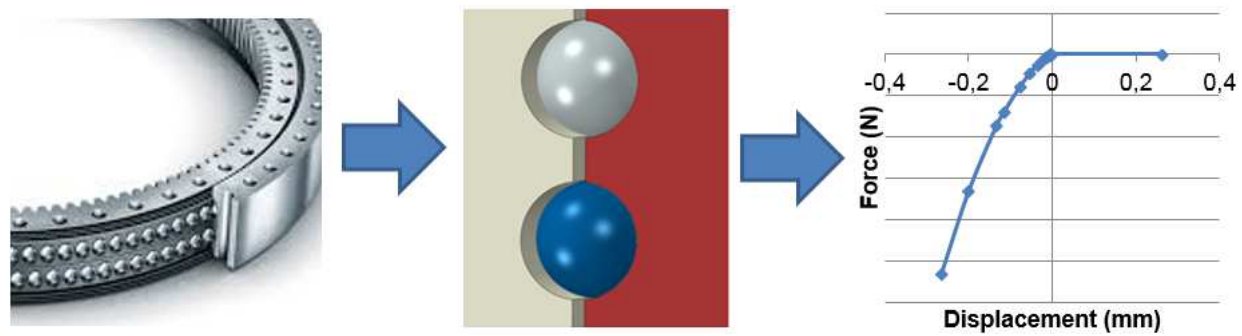

Fig. 6 Nonlinear spring constant for balls

The global finite element model must reflect the overall response of the structure in order to accurately evaluate it. Dynamic firing forces (plume force) are applied on the global finite element model. Transient dynamic response of the structure under operational loads was compared to the finite element solution by using displacement measurements at different locations. In addition to that, strain gages were placed at several locations (Fig. 7) and overall stress distribution of the finite element solution was verified. In Fig. 8 and Fig. 9 FEM results and test measurements are compared. Normalizations were performed against maximum sensor readings. More information concerning dynamic finite element analysis of the cradle system can be found in [6].

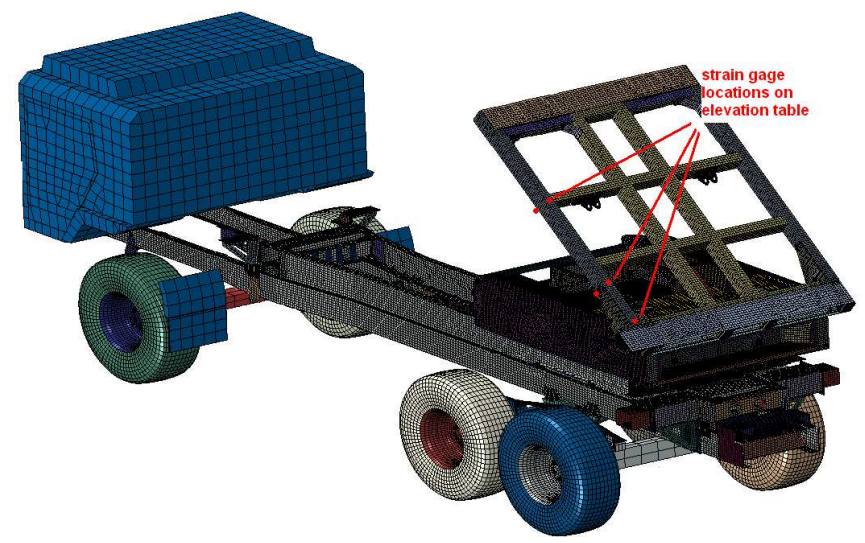

Fig. 7 Sensor locations 


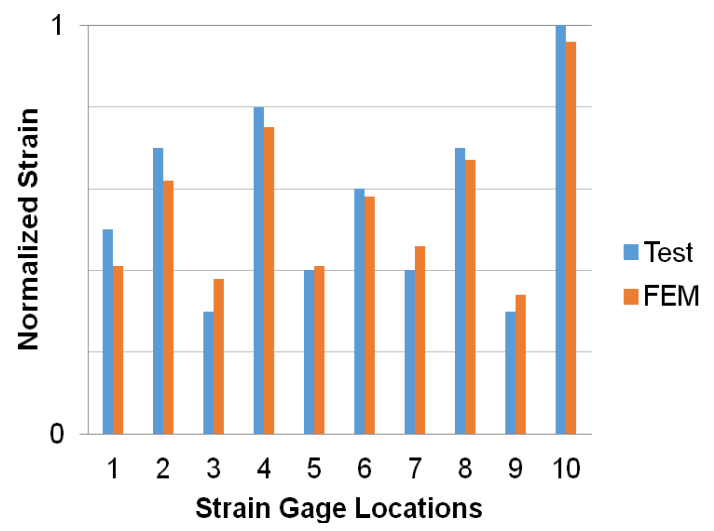

Fig. 8 Normalized test and FEM strains at different strain gage locations

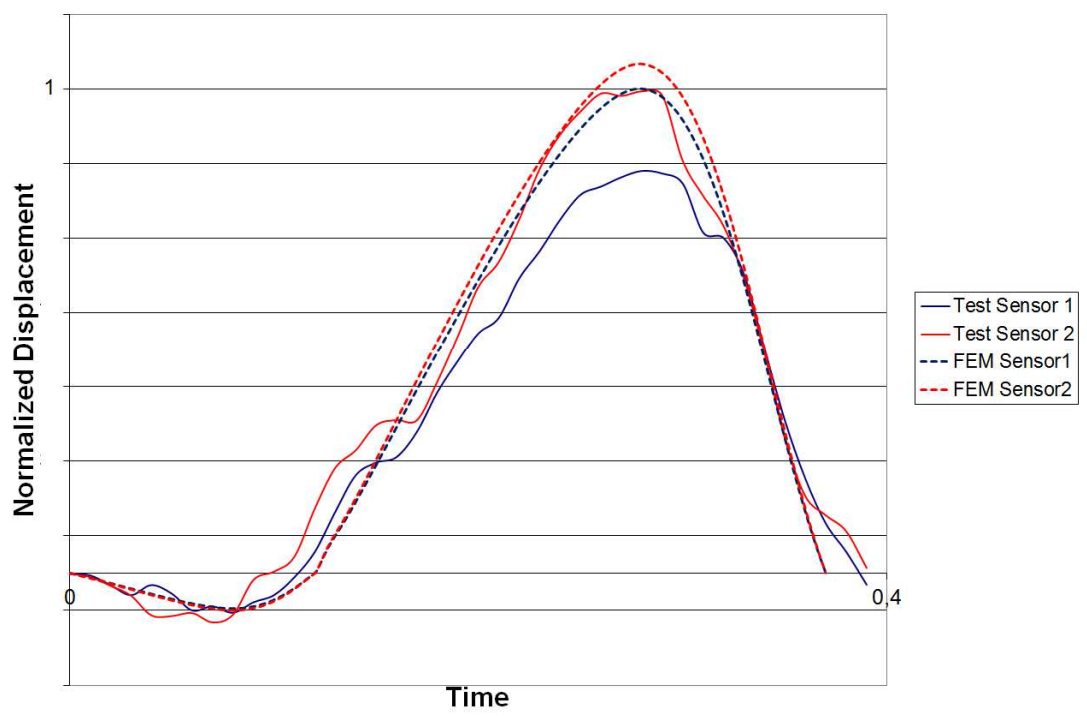

Fig. 9 Normalized test and FEM displacements

\section{Sub-Model}

In real life, structures are likely to have semi-elliptical surface or circular cracks around welds. Cracks can be positioned at different locations on the weld geometry and at different angles at a location. Cracks may exist inherently due to welding process in the structure or they may initiate due to operational loading on the structure. Under operational loads, these cracks could grow in an unstable manner and cause total failure. It is also possible to have fatigue failure under repetitive loading (i.e. crack propagation at each load cycle) but that case is not addressed in this paper. In this study, maximum allowable crack size, which would not spread in an unstable manner is investigated at critical stress locations and possible crack zones. 
Fracture analysis is made within the framework of linear elastic fracture mechanics [7]. Cracks in the structure are modelled by embedding them in a region meshed by using solid elements. Therefore sub-models are converted to three dimensional elements in the crack vicinity. Semi-elliptical surface cracks are investigated in this study. Along crack front, special elements are created for stress intensity factor calculations. Collapsed wedge like quadratic elements with middle nodes positioned at quarter point from element vertex are used in order to capture square root crack tip singularity [8].

Crack models with different mesh densities are created and results are compared. In Fig. 10, only crack zone of sub models in different mesh densities can be seen. Element density is found for which stress intensity factor does not change significantly over crack front (see Fig. 11). In fine model overall element size of $0.14 \mathrm{~mm}$ with 46 elements in crack front and 16 elements in circumferential direction, in coarse model overall element size of $0.25 \mathrm{~mm}$ with 24 elements in crack front and 8 elements in circumferential direction were used. Angle $\Phi$ is measured from free surface of crack front.
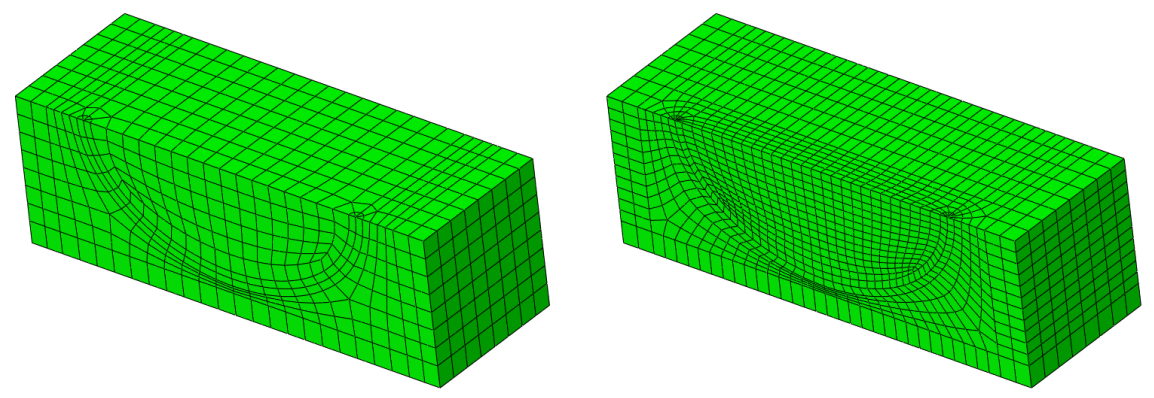

Fig. 10 Coarse and fine crack models

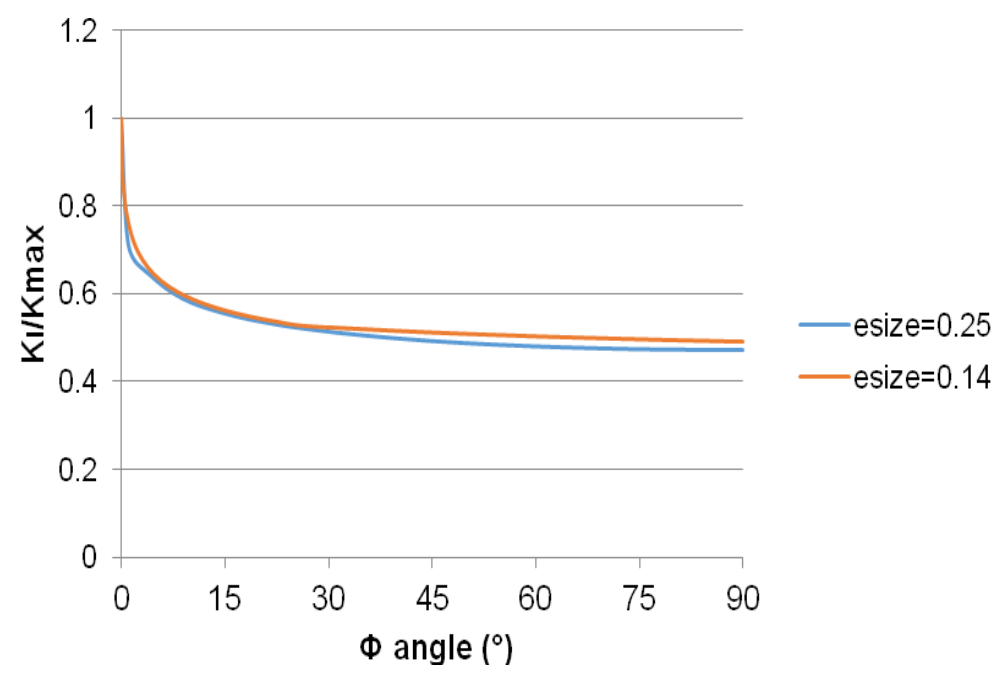

Fig. 11 Stress intensity factor for coarse and fine models 


\section{Stress Intensity Factor Calculations}

ABAQUS ${ }^{\circledR}$ offers different types of contour integrals for onset of crack propagation in fracture mechanics analysis. It uses $J$ integral calculation if not a different type is requested by the user. If material is defined as linear, stress intensity factors can be related to $J$ integral [3].

$J$ integral for a linear elastic material can be related to stress intensity factor through Eq. (1) in which $\boldsymbol{B}$ is defined as pre-logarithmic energy factor matrix.

$$
\boldsymbol{K}=\left[K_{\mathrm{I}}, K_{\mathrm{II}}, K_{\mathrm{III}}\right]^{\mathrm{T}} .
$$

Generally $J$ integral can be written as:

$$
J=\frac{1}{8 \pi}\left[\begin{array}{l}
K_{\mathrm{I}} B_{11}^{-1} K_{\mathrm{I}}+2 K_{\mathrm{I}} B_{12}^{-1} K_{\mathrm{II}}+2 K_{\mathrm{I}} B_{13}^{-1} K_{\mathrm{III}} \\
+\left(\text { terms not involving } K_{\mathrm{I}}\right)
\end{array}\right],
$$

I, II, III refers to 1, 2, 3 when components of $\boldsymbol{B}$ are indicated. $J$ integral for an auxiliary pure Mode I crack tip field with $k_{1}$ as stress intensity factor can be defined as:

$$
J_{\text {aux }}^{\mathrm{I}}=\frac{1}{8 \pi} k_{1} B_{11}^{-1} K_{\mathrm{I}} .
$$

When auxiliary field is added to actual field:

$$
J_{\text {tot }}^{\mathrm{I}}=\frac{1}{8 \pi}\left[\begin{array}{l}
\left(K_{\mathrm{I}}+k_{1}\right) B_{11}^{-1}\left(K_{\mathrm{I}}+k_{1}\right)+2\left(K_{\mathrm{I}}+k_{1}\right) B_{12}^{-1} K_{\mathrm{II}} \\
+2\left(K_{\mathrm{I}}+k_{1}\right) B_{13}^{-1} K_{\mathrm{III}}+\left(\text { terms not involving } K_{\mathrm{I}} \text { or } k_{1}\right)
\end{array}\right] .
$$

Since the terms not involving $K_{1}$ or $k_{1}$ are equal in total field and actual field, interaction integral can be defined as:

$$
J_{\text {int }}^{\mathrm{I}}=J_{\text {tot }}^{\mathrm{I}}-J-J_{\text {aux }}^{\mathrm{I}}=\frac{k_{1}}{4 \pi}\left[B_{11}^{-1} K_{\mathrm{I}}+B_{12}^{-1} K_{\mathrm{II}}+B_{13}^{-1} K_{\mathrm{III}}\right] .
$$

When the calculations are also done for Mode II and III, an interaction integral can be given as Eq. (6).

$$
J_{\text {aux }}^{\alpha}=\frac{k_{\alpha}}{4 \pi} B_{\alpha \beta}^{-1} K_{\beta} .
$$

Above equation can be given as below by assigning unit values to $k_{\alpha}$.

$$
\begin{gathered}
\boldsymbol{K}=4 \pi \boldsymbol{B} \boldsymbol{J}_{\text {int }}, \\
\boldsymbol{J}_{\text {int }}=\left[J_{\text {int }}^{\mathrm{I}}, J_{\text {int }}^{\mathrm{II}}, J_{\text {int }}^{\mathrm{III}}\right]^{\mathrm{T}} .
\end{gathered}
$$

The software uses an interaction integral method in order to compute stress intensity factors in mixed mode for a calculated $J$ integral value. This extraction method can be used for isotropic and anisotropic linear materials. Details of calculation method can be found in [3]. 


\section{Results from three different crack locations}

Cracks were modelled in three different locations at a weld determined by previous design experiences. Connections were examined and positions in which the flaws are most likely to occur were determined and used in further design studies. Cracks at weld toe are commonly seen flaws in welded structures. These cracks initiate from flaws left behind welding process.

In this study, some results pertaining to one particular weld, which is found to be critical are given. At this weld, three different types of crack models namely semi-elliptical surface crack at weld toe outer surface, semi-elliptical surface crack at weld toe inner surface, and also an embedded circular crack are implemented. Within the context of this study, for each crack model, stress intensity factors for all three modes are obtained. Also different semi-elliptical crack aspect ratios are modelled and results are found. For brevity, these results are not included here, but they could be found in [9].
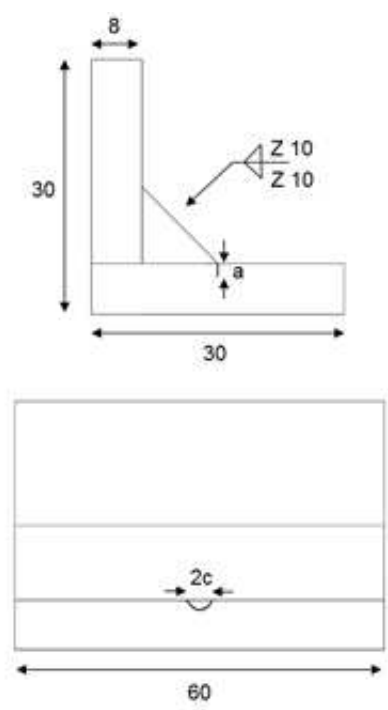

(a)
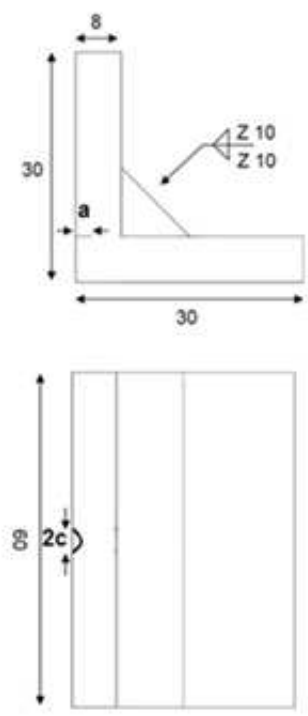

(b)
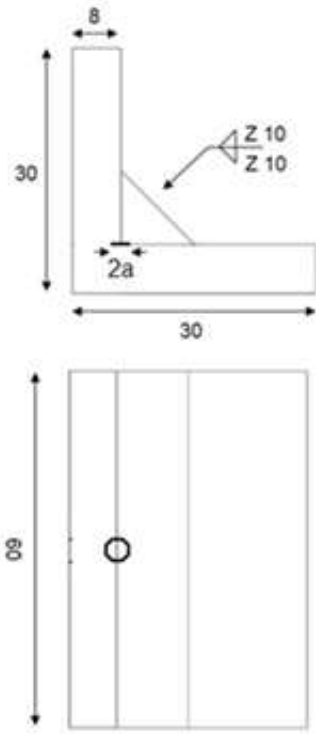

(c)

Fig. 12 Dimensions of solid sections of sub model (a) semi elliptical surface crack at weld toe outer surface, (b) semi elliptical surface crack at weld toe inner surface, (c) embedded circular crack

For the given load case and geometry, and for an aspect ratio of $\mathrm{a} / \mathrm{c}=0.5$, stress intensity factor comparison for three different crack configurations are given in Fig.15. The comparison is based on mode I stress intensity factor, which is the dominant one. Crack size is normalized against most critical condition's crack size, which belongs to semi-elliptical surface crack at weld toe. (Most critical crack size refers to the size at which mode I stress intensity factor equals the plain strain fracture toughness of the material, which is steel.) Among three crack cases, semi-elliptical crack at weld toe gives highest stress intensity factor for this load case and geometry. 


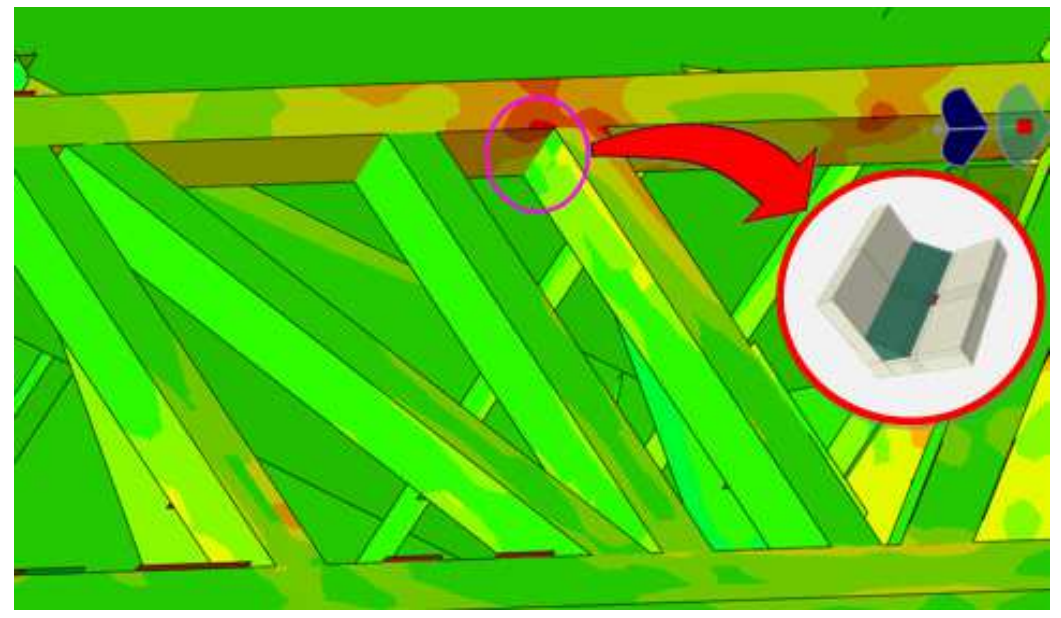

Fig. 13 Critical location, which is examined in this study
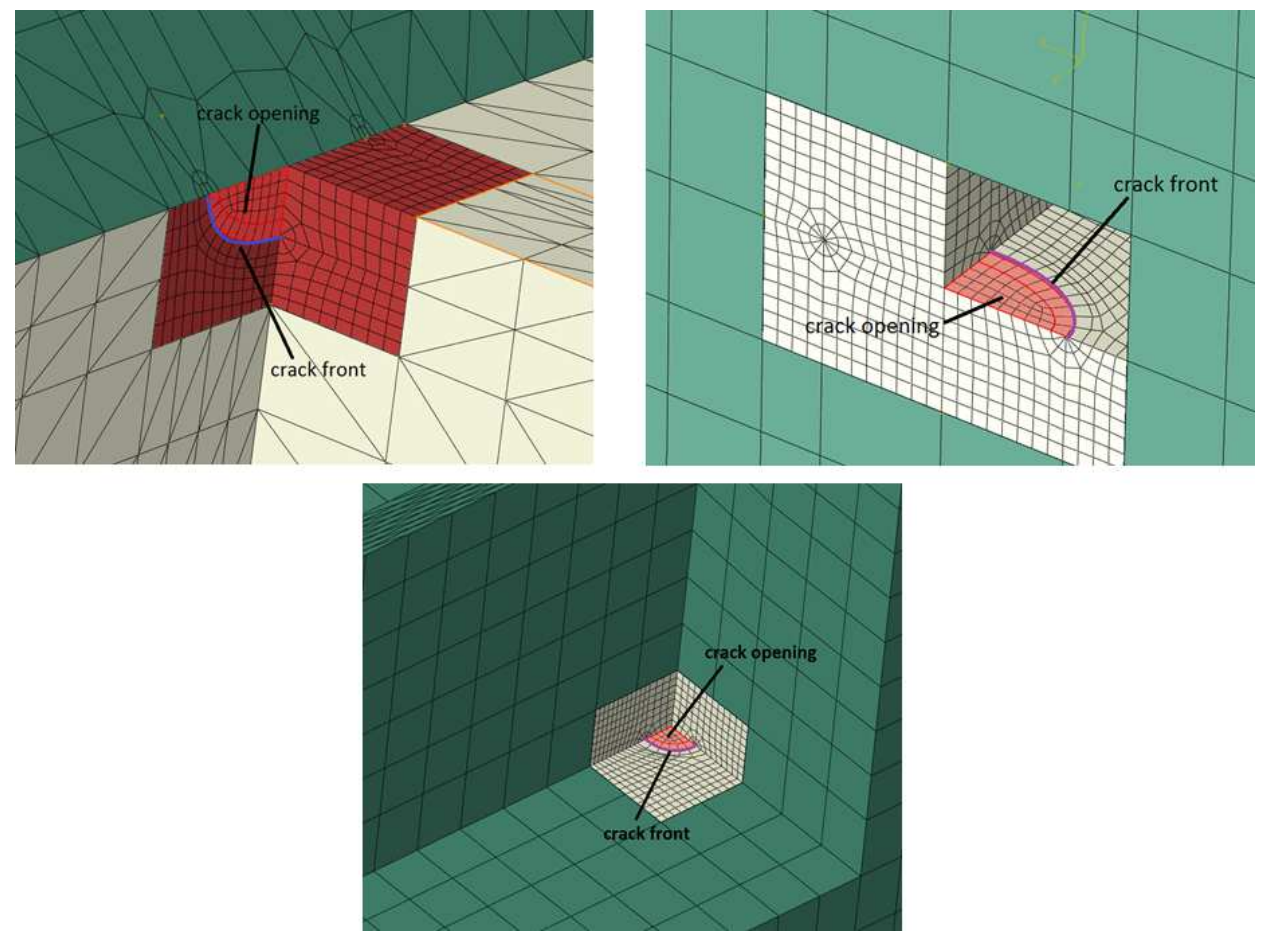

Fig.14 Sub model crack details

Stress intensity factor value is checked against fracture toughness value of the material. In general loading conditions, structures are subjected to mixed mode loading. However in most of the cases, mode I stress intensity factor is dominant over other modes namely mode II and III. Therefore it is reasonably practical to check mode I stress intensity factor. 


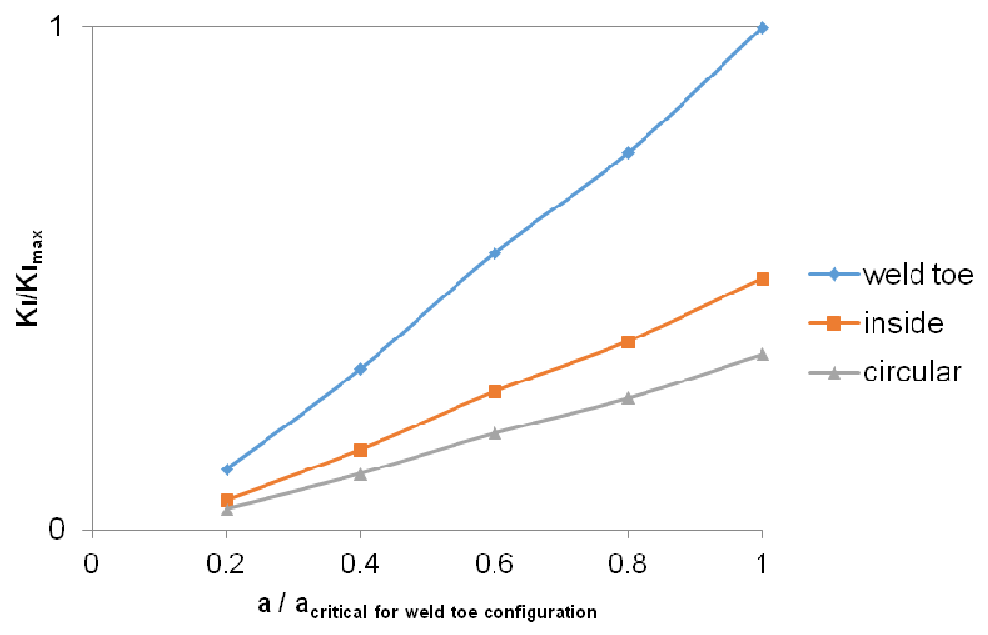

Fig.15. Comparison of three crack configurations

\section{Discussion}

Critical systems that are subjected to high amplitude dynamic and repeated loading must be subjected to thorough structural evaluations. Design of such systems can not be solely based on yielding or tensile strength of material. The system will most likely have geometric imperfections. These imperfections may be inherent due to manufacturing or occur after some loading. Such a structure with imperfections or flaws must be investigated carefully. Fracture mechanics is used for damage-tolerant design of the structure with imperfections.

Limiting value of materials up to, which they can be loaded can be much less in case of fracture mechanics. Therefore, a design cycle including fracture mechanics analysis must be implemented. To accomplish that, finite element method is used. Modelling methodology was presented here and a study of a connection under one load case was given. Fracture mechanics analysis is important in design study for structural integrity assessment, and it must be included in the design process.

Sub-modelling method is an appropriate way of modelling cracks. When global finite element models are large, it is impractical to include details such as crack in the model; therefore, sub-modelling is applied. Global finite element model is solved once for one load case and various sub-models can be created from the same global model solution. It allows different configurations to be studied at the same location.

In this work, a methodology for modelling cracks in very big and complicated systems was proposed besides numerical results of investigated cases for critical crack size. The launcher system is checked using non-destructive testing methods for critical flaw size around welded connection, which is found to be critical location. Damage-tolerant and safe life design concepts were introduced briefly. The work presented here is a part of the safe life design approach for the design and manufacturing of a launcher system. Critical locations of welds in the system were checked after repeated loading of firings for presence of any cracks. After these controls, no cracks were detected due to manufacturing or operational conditions until the time when this document was prepared. 


\section{Acknowledgement}

The authors would like to acknowledge the contribution of Roketsan Missiles Ind.

\section{References}

[1] US Army Weapon Systems Handbook. Washington: OASA, 1999.

[2] WANG, C.H. Introduction to Fracture Mechanics. DSTO Aeronautical and Maritime Research Laboratory Melbourne, DSTO-GD-0103, 1996.

[3] ABAQUS ${ }^{\circledR}$ Documentation v6.12.

[4] DIAMANTOUDIS, A.Th. and LABEAS, G.N. Stress Intensity Factors of Semi-elliptical Surface Cracks in Pressure Vessels by Global-local Finite Element Methodology. Engineering Fracture Mechanics, 2005, vol. 72, no. 9, p.1299-1312. DOI 10.1016/j.engfracmech.2004.10.004.

[5] GIGLIO M. and MANES A. Crack Propagation on Helicopter Panel: Experimental Test and Analysis. Engineering Fracture Mechanics, 2008, vol. 75, no. 3-4, p. 866-879. DOI 10.1016/j.engfracmech.2007.02.024.

[6] ACAR, B., YETGIN, A. and KADIOGLU S. Dynamic Finite Element Analysis of a Cradle System. In 84 ${ }^{\text {th }}$ Shock and Vibration Symposium, 2013.

[7] ANDERSON, T.L. Fracture Mechanics: Fundamentals and Applications. $3^{\text {rd }}$ edition. Boca Raton: Taylor \& Francis, 2005.

[8] ACAR, B. Finite element analysis of fracture mechanics problems [Master's Thesis], Ankara: Middle East Technical University, 1997.

[9] YETGIN, A. Fracture Analysis of Welded Connections [Master Thesis]. Ankara: Middle East Technical University, 2013. 